\title{
Global Existence of Solutions to the 2D Incompressible Generalized Liquid Crystal Flow
}

\author{
Mingxuan Zhu \\ School of Mathematical Sciences, Xiamen University, Xiamen, China \\ Correspondence should be addressed to Mingxuan Zhu; zhumingxuan587@gmail.com
}

Received 11 April 2014; Accepted 21 April 2014; Published 6 May 2014

Academic Editor: Yonghui Xia

Copyright ( 2014 Mingxuan Zhu. This is an open access article distributed under the Creative Commons Attribution License, which permits unrestricted use, distribution, and reproduction in any medium, provided the original work is properly cited.

We consider the global existence of solutions to the $2 \mathrm{D}$ incompressible generalized liquid crystal flow. It is proved that the local solution exists globally with $\beta=0, \alpha \geq 2$.

\section{Introduction}

In this paper, we consider the following 2D liquid crystal flow:

$$
\begin{gathered}
u_{t}+u \cdot \nabla u+\nabla p+\Lambda^{2 \alpha} u=-\nabla d \cdot \Delta d, \\
d_{t}+u \cdot \nabla d+\Lambda^{2 \beta} d=-f(d), \\
\operatorname{div} u=0, \\
\left.(u, d)\right|_{t=0}=\left(u_{0}, d_{0}\right),
\end{gathered}
$$

where $\alpha \geq 0, \beta \geq 0$ are real parameters and $u$ is the velocity, $d$ is a vectorial function modeling the orientation of the crystal molecules, and $p$ is the scalar pressure. Here $f(d):=\left(|d|^{2}-\right.$ 1) $d$ and $\Lambda=(-\Delta)^{1 / 2}$ is defined in terms of Fourier transform by

$$
\widehat{\Lambda f}(\xi)=|\xi| \widehat{f}(\xi) .
$$

When $\alpha=\beta=1$, it has been shown that (1)-(4) has unique global weak and smooth solutions [1-3]. In [4], global regularity for this system with mixed partial viscosity is proved. Some regularity criteria are established for the system with zero dissipation in [5].

The aim of this paper is to establish the following global regularity for the $2 \mathrm{D}$ liquid crystal model with fractional diffusion.

Theorem 1. Assume $\left(u_{0}, d_{0}\right) \in H^{3}\left(\mathbb{R}^{2}\right) \times H^{4}\left(\mathbb{R}^{2}\right)$. Let $(u, d)$ be the local strong solution to the problem (1)-(4). If $\alpha$ and $\beta$ satisfy $\beta=0, \alpha \geq 2$, then the $2 D$ liquid crystal model has a unique global classical solution $(u, d)$ satisfying

$$
\begin{gathered}
u \in L^{\infty}\left(0, T ; H^{3}\left(\mathbb{R}^{2}\right)\right), \quad u \in L^{2}\left(0, T ; H^{3+\alpha}\left(\mathbb{R}^{2}\right)\right), \\
d \in L^{\infty}\left(0, T ; H^{4}\left(\mathbb{R}^{2}\right)\right) .
\end{gathered}
$$

Remark 2. This work is partially motivated by the recent progress on the 2D incompressible MHD system with fractional diffusion; we refer to [6-10] and references therein. In [7], Tran et al. obtained the global regularity of 2D GMHD equations for the following three cases: (1) $\alpha \geq 1, \beta \geq 1$; (2) $0 \leq \alpha<1 / 2,2 \alpha+\beta>2$; (3) $\alpha \geq 2, \beta=0$. Combining them with the result in [10], we know that if $\alpha+\beta \geq 2$, 2D incompressible MHD system with fractional diffusion possesses a global smooth solution. Fan et al. [8] proved the global existence of smooth solutions with $\alpha>0, \beta=1$. Global regularity for the case $\alpha=0, \beta>1$ was established by Jiu and Zhao [9] which improves the result in [6]. Very recently, the authors improved the case $\alpha=0, \beta>1$ for the 2D liquid crystal model in [11].

\section{Proof of Theorem 1}

It is sufficient to prove Theorem 1 with $\alpha=2, \beta=0$.

We will prove Theorem 1 if we can demonstrate the boundedness of $\|u\|_{H^{3}}^{2}+\|d\|_{H^{4}}^{2}$. In order to reach our purpose, we will show this by contradiction: assume

$$
\lim _{t \rightarrow T} \sup \|u\|_{H^{3}}^{2}+\|d\|_{H^{4}}^{2}=\infty
$$


for some finite time $T>0$. Our thought is that when $T_{0}$ is close enough to $T,\|u\|_{H^{3}}^{2}+\|d\|_{H^{4}}^{2}$ remains uniformly bounded for $T_{0}<t<T$ under such assumption, thus reaching a contradiction.

First, we do $L^{2}$ estimate for $d$. Multiplying (2) by $d$ and using (3), after integration by parts, we see that

$$
\frac{1}{2} \frac{d}{d t}\|d\|_{L^{2}}^{2}+\|d\|_{L^{4}}^{4}=\|d\|_{L^{2}}^{2} .
$$

By using the Gronwall inequality, we have

$$
\|d\|_{L^{2}}+\int_{0}^{T}\|d\|_{L^{4}}^{4} d \tau \leq C .
$$

Then, we will show the $L^{2}$ estimate for $u$ and $\nabla d$. Multiplying (1) and (2) by $u$ and $-\Delta d$, respectively, we find that

$$
\begin{aligned}
\frac{1}{2} \frac{d}{d t} & \left(\|u\|_{L^{2}}^{2}+\|\nabla d\|_{L^{2}}^{2}\right)+\left\|\Lambda^{2} u\right\|_{L^{2}}^{2} \\
& =-\int_{\mathbb{R}^{2}} \nabla f(d) \nabla d d x \\
& \leq-3 \int_{\mathbb{R}^{2}}|d|^{2}|\nabla d|^{2} d x+\|\nabla d\|_{L^{2}}^{2} .
\end{aligned}
$$

Thanks to Gronwall's inequality and (9), we have

$$
\|u\|_{L^{2}}^{2}+\|\nabla d\|_{L^{2}}^{2}+\int_{0}^{T}\left\|\Lambda^{2} u\right\|_{L^{2}}^{2} d \tau \leq C,
$$

which means $\nabla u \in L^{2}(0, T ; \mathrm{BMO})$.

The $H^{1}$ estimate for $u$ and $H^{2}$ estimate for $d$ will be shown as follows. Multiplying (1) by $\Delta u$, applying $\Delta$ to (2), multiplying by $\Delta d$, and then summing them up, we obtain

$$
\begin{aligned}
\frac{1}{2} \frac{d}{d t}( & \left.\|\nabla u\|_{L^{2}}^{2}+\|\Delta d\|_{L^{2}}^{2}\right)+\|\Delta \nabla u\|_{L^{2}}^{2} \\
\leq & \int_{\mathbb{R}^{2}} \nabla d \cdot \Delta d \cdot \Delta u-\Delta(u \cdot \nabla d) \cdot \Delta d \\
& -\Delta f(d) \cdot \Delta d d x \\
\leq & C\|\Delta d\|_{L^{2}}^{2}\|\nabla u\|_{L^{\infty}} \\
& +\int_{\mathbb{R}^{2}}-3|d|^{2}|\Delta d|^{2}-d|\nabla d|^{2} \Delta d+|\Delta d|^{2} d x \\
\leq & C\|\Delta d\|_{L^{2}}^{2}\|\nabla u\|_{L^{\infty}}-2\|d \Delta d\|_{L^{2}}^{2}+C\|\nabla d\|_{L^{4}}^{4}+\|\Delta d\|_{L^{2}}^{2} \\
\leq & C\|\Delta d\|_{L^{2}}^{2}\left(\|\nabla u\|_{L^{\infty}}+1\right)-2\|d \Delta d\|_{L^{2}}^{2} \\
\leq & C\|\Delta d\|_{L^{2}}^{2}\left(\|\nabla u\|_{L^{\infty}}+1\right) .
\end{aligned}
$$

Let us introduce the following commutator and bilinear estimates established in $[12,13]$ :

$$
\begin{aligned}
& \left\|\Lambda^{s}(f g)-f \Lambda^{s} g\right\|_{L^{p}} \\
& \quad \leq C\left(\|\nabla f\|_{L^{p_{1}}}\left\|\Lambda^{s-1} g\right\|_{L^{q_{1}}}+\|g\|_{L^{p_{1}}}\left\|\Lambda^{s} f\right\|_{L^{q_{1}}}\right), \\
& \quad\left\|\Lambda^{s}(f g)\right\|_{L^{p}} \\
& \quad \leq C\left(\|f\|_{L^{p_{1}}}\left\|\Lambda^{s} g\right\|_{L^{q_{1}}}+\left\|\Lambda^{s} f\right\|_{L^{p_{2}}}\|g\|_{L^{q_{2}}}\right),
\end{aligned}
$$

with $s>0$ and $1 / p=1 / p_{1}+1 / q_{1}=1 / p_{2}+1 / q_{2}$.
Now, we do the $H^{2}$ estimate for $u$ and $H^{3}$ estimate for $d$. Applying $\Lambda^{2}$ to (1), multiplying by $\Lambda^{2} u$, and dealing with (2) in the same way by $\Lambda^{3}$ and $\Lambda^{3} d$, after summing them up, we have

$$
\begin{gathered}
\frac{1}{2} \frac{d}{d t}\left(\left\|\Lambda^{2} u\right\|_{L^{2}}^{2}+\left\|\Lambda^{3} d\right\|_{L^{2}}^{2}\right)+\left\|\Lambda^{4} u\right\|_{L^{2}}^{2} \\
=\int_{\mathbb{R}^{2}}-\Lambda^{2}(u \cdot \nabla u) \Lambda^{2} u-\Lambda^{2}(\nabla d \cdot \Delta d) \Lambda^{2} u \\
\quad-\Lambda^{3}(u \cdot \nabla d) \Lambda^{3} d-\Lambda^{3} f(d) \Lambda^{3} d d x \\
=: I_{1}+I_{2}+I_{3}+I_{4} .
\end{gathered}
$$

Using Hölder's inequality, Gagliardo-Nirenberg inequality, Young's inequality, and (13), we have the following estimates:

$$
\begin{aligned}
\left|I_{1}\right| & =\left|\int_{\mathbb{R}^{2}}\left(\Lambda^{2}(u \cdot \nabla u)-u \cdot \nabla \Lambda^{2} u\right) \Lambda^{2} u d x\right| \\
& \leq C\|\nabla u\|_{L^{\infty}}\left\|\Lambda^{2} u\right\|_{L^{2}}^{2}, \\
\left|I_{2}\right| & \leq C\left\|\Lambda^{4} u\right\|_{L^{2}}\|\nabla d\|_{L^{4}}\left\|\Lambda^{2} d\right\|_{L^{4}} \\
& \leq C\left\|\Lambda^{4} u\right\|_{L^{2}}\|\nabla d\|_{L^{2}}\left\|\Lambda^{3} d\right\|_{L^{2}} \\
& \leq \frac{1}{4}\left\|\Lambda^{4} u\right\|_{L^{2}}^{2}+\left\|\Lambda^{3} d\right\|_{L^{2}}^{2}, \\
\left|I_{3}\right| & \leq C\left|\int_{\mathbb{R}^{2}} \Lambda^{3}(u \cdot \nabla d) \Lambda^{3} d-u \cdot \nabla \Lambda^{3} d \Lambda^{3} d d x\right| \\
& \leq C \int_{\mathbb{R}^{2}}\left|\Lambda^{3} u\right||\Lambda d|\left|\Lambda^{3} d\right|+\left|\Lambda^{2} u\right|\left|\Lambda^{2} d\right|\left|\Lambda^{3} d\right| \\
& +I I_{1}+I I_{2}+I I_{3} .
\end{aligned}
$$

Now we estimate $I I_{1}, I I_{2}$, and $I I_{3}$ one by one:

$$
\begin{aligned}
& I I_{1} \leq C\left\|\Lambda^{3} u\right\|_{L^{4}}\|\Lambda d\|_{L^{4}}\left\|\Lambda^{3} d\right\|_{L^{2}} \\
& \leq C\left\|\Lambda^{2} u\right\|_{L^{2}}^{1 / 4}\left\|\Lambda^{4} u\right\|_{L^{2}}^{3 / 4}\|\Lambda d\|_{L^{2}}^{3 / 4}\left\|\Lambda^{3} d\right\|_{L^{2}}^{5 / 4} \\
& \leq \frac{1}{8}\left\|\Lambda^{4} u\right\|_{L^{2}}^{2}+C\left\|\Lambda^{2} u\right\|_{L^{2}}^{2 / 5}\left\|\Lambda^{3} d\right\|_{L^{2}}^{2}, \\
& I I_{2} \leq C\left\|\Lambda^{2} u\right\|_{L^{4}}\left\|\Lambda^{2} d\right\|_{L^{4}}\left\|\Lambda^{3} d\right\|_{L^{2}} \\
& \leq C\left\|\Lambda^{2} u\right\|_{L^{2}}^{3 / 4}\left\|\Lambda^{4} u\right\|_{L^{2}}^{1 / 4}\|\Lambda d\|_{L^{2}}^{1 / 4}\left\|\Lambda^{3} d\right\|_{L^{2}}^{7 / 4} \\
& \leq \frac{1}{8}\left\|\Lambda^{4} u\right\|_{L^{2}}^{2}+\left\|\Lambda^{2} u\right\|_{L^{2}}^{6 / 7}\left\|\Lambda^{3} d\right\|_{L^{2}}^{2}, \\
& I I_{3} \leq C\|\nabla u\|_{L^{\infty}}\left\|\Lambda^{3} d\right\|_{L^{2}}^{2},
\end{aligned}
$$


Abstract and Applied Analysis

3

$$
\begin{aligned}
I_{4}= & \left\|\Lambda^{3} d\right\|_{L^{2}}^{2}-\int_{\mathbb{R}^{2}} \Lambda^{3}\left(|d|^{2} d\right) \Lambda^{3} d \\
\leq & \left\|\Lambda^{3} d\right\|_{L^{2}}^{2}-3\left\|d \Lambda^{3} d\right\|_{L^{2}}^{2} \\
& +C \int_{\mathbb{R}^{2}}\left|\Lambda^{2} d\right||\Lambda d||d|\left|\Lambda^{3} d\right|+C \int_{\mathbb{R}^{2}}|\Lambda d|^{3}\left|\Lambda^{3} d\right| \\
= & :\left\|\Lambda^{3} d\right\|_{L^{2}}^{2}-3\left\|d \Lambda^{3} d\right\|_{L^{2}}^{2}+K_{1}+K_{2} .
\end{aligned}
$$

$K_{1}$ and $K_{2}$ can be estimated as follows:

$$
\begin{aligned}
K_{1} & \leq C\|\Lambda d\|_{L^{4}}\left\|\Lambda^{2} d\right\|_{L^{4}}\left\|d \Lambda^{3} d\right\|_{L^{2}} \\
& \leq C\|\Lambda d\|_{L^{2}}^{1 / 4}\left\|\Lambda^{3} d\right\|_{L^{2}}^{3 / 4}\|\Lambda d\|_{L^{2}}^{3 / 4}\left\|\Lambda^{3} d\right\|_{L^{2}}^{1 / 4}\left\|d \Lambda^{3} d\right\|_{L^{2}} \\
& \leq C\left\|\Lambda^{3} d\right\|_{L^{2}}^{2}+3\left\|d \Lambda^{3} d\right\|_{L^{2}}^{2}, \\
K_{2} & \leq C\|\Lambda d\|_{L^{6}}^{3}\left\|\Lambda^{3} d\right\|_{L^{2}} \\
& \leq C\left(\|\Lambda d\|_{L^{2}}^{2 / 3}\left\|\Lambda^{3} d\right\|_{L^{2}}^{1 / 3}\right)^{3}\left\|\Lambda^{3} d\right\|_{L^{2}} \leq C\left\|\Lambda^{3} d\right\|_{L^{2}}^{2} .
\end{aligned}
$$

Combining $K_{1}$ and $K_{2}$, we have

$$
I_{4} \leq C\left\|\Lambda^{3} d\right\|_{L^{2}}^{2} .
$$

Summing all the above estimates to (14), we obtain

$$
\begin{aligned}
& \frac{d}{d t}\left(\left\|\Lambda^{2} u\right\|_{L^{2}}^{2}+\left\|\Lambda^{3} d\right\|_{L^{2}}^{2}\right)+\left\|\Lambda^{4} u\right\|_{L^{2}}^{2} \\
& \quad \leq C\left(\|\nabla u\|_{L^{\infty}}+\left\|\Lambda^{2} u\right\|_{L^{2}}\right)\left(\left\|\Lambda^{2} u\right\|_{L^{2}}^{2}+\left\|\Lambda^{3} d\right\|_{L^{2}}^{2}\right) .
\end{aligned}
$$

Now, we will show the $H^{3}$ estimate for $u$ and $H^{4}$ estimate for $d$. Applying $\Lambda^{3}$ to (1), multiplying by $\Lambda^{3} u$, and dealing with (2) in the same way by $\Lambda^{4}$ and $\Lambda^{4} d$, after summing them up, we have

$$
\begin{aligned}
\frac{1}{2} \frac{d}{d t} & \left(\left\|\Lambda^{3} u\right\|_{L^{2}}^{2}+\left\|\Lambda^{4} d\right\|_{L^{2}}^{2}\right)+\left\|\Lambda^{5} u\right\|_{L^{2}}^{2} \\
= & \int_{\mathbb{R}^{2}}-\Lambda^{3}(u \cdot \nabla u) \Lambda^{3} u-\Lambda^{3}(\nabla d \cdot \Delta d) \Lambda^{3} u \\
& \quad-\Lambda^{4}(u \cdot \nabla d) \Lambda^{4} d-\Lambda^{4} f(d) \Lambda^{4} d d x \\
= & J_{1}+J_{2}+J_{3}+J_{4} .
\end{aligned}
$$

Using Hölder's inequality, Gagliardo-Nirenberg inequality, Young's inequality, and (13), we have the following estimates:

$$
\begin{gathered}
\left|J_{1}\right| \leq C\|\nabla u\|_{L^{\infty}}\left\|\Lambda^{3} u\right\|_{L^{2}}^{2}, \\
\left|J_{2}\right| \leq C \int_{\mathbb{R}^{2}}|\Lambda(\nabla d \Delta d)|\left|\Lambda^{5} u\right| d x \leq\|\Lambda(\nabla d \cdot \Delta d)\|_{L^{2}}\left\|\Lambda^{5} u\right\|_{L^{2}} \\
\leq C\left\|\Lambda^{5} u\right\|_{L^{2}}\left(\|\Delta d\|_{L^{4}}^{2}+\|\Lambda d\|_{L^{4}}\left\|\Lambda^{3} d\right\|_{L^{4}}\right) \\
\leq C\left\|\Lambda^{5} u\right\|_{L^{2}}\left(\|\Lambda d\|_{L^{2}}\left\|\Lambda^{4} d\right\|_{L^{2}}+\|\Lambda d\|_{L^{2}}^{5 / 6}\left\|\Lambda^{4} d\right\|_{L^{2}}^{1 / 6}\right. \\
\left.\times\|\Lambda d\|_{L^{2}}^{1 / 6}\left\|\Lambda^{4} d\right\|_{L^{2}}^{5 / 6}\right)
\end{gathered}
$$

$$
\begin{gathered}
\leq C\left\|\Lambda^{4} d\right\|_{L^{2}}^{2}+\frac{1}{4}\left\|\Lambda^{5} u\right\|_{L^{2}}^{2}, \\
\left|J_{3}\right|=C\left|\int_{\mathbb{R}^{2}}\left(\Lambda^{4}(u \cdot \nabla d)-u \cdot \nabla \Lambda^{4} d\right) \Lambda^{4} d d x\right| \\
\leq C \int_{\mathbb{R}^{2}}\left|\Lambda^{4} u\right||\nabla d|\left|\Lambda^{4} d\right|+\left|\Lambda^{3} u\right|\left|\Lambda^{2} d\right|\left|\Lambda^{4} d\right| \\
\quad+\left|\Lambda^{2} u\right|\left|\Lambda^{3} d\right|\left|\Lambda^{4} d\right|+|\Lambda u|\left|\Lambda^{4} d\right|^{2} d x \\
=: J_{31}+J_{32}+J_{33}+J_{34} .
\end{gathered}
$$

Now we estimate $J_{31}, J_{32}, J_{33}$, and $J_{34}$ one by one:

$$
\begin{aligned}
\left|J_{31}\right| & \leq C\left\|\Lambda^{4} u\right\|_{L^{4}}\|\Lambda d\|_{L^{4}}\left\|\Lambda^{4} d\right\|_{L^{2}} \\
& \leq C\left\|\Lambda^{2} u\right\|_{L^{2}}^{1 / 6}\left\|\Lambda^{5} u\right\|_{L^{2}}^{5 / 6}\|\Lambda d\|_{L^{2}}^{5 / 6}\left\|\Lambda^{4} d\right\|_{L^{2}}^{7 / 6} \\
& \leq \frac{1}{8}\left\|\Lambda^{5} u\right\|_{L^{2}}^{2}+C\left\|\Lambda^{2} u\right\|_{L^{2}}^{3 / 7}\left\|\Lambda^{4} d\right\|_{L^{2}}^{2}, \\
\left|J_{32}\right| & \leq C\left\|\Lambda^{3} u\right\|_{L^{4}}\left\|\Lambda^{2} d\right\|_{L^{4}}\left\|\Lambda^{4} d\right\|_{L^{2}} \\
& \leq C\left\|\Lambda^{2} u\right\|_{L^{2}}^{1 / 2}\left\|\Lambda^{5} u\right\|_{L^{2}}^{1 / 2}\|\Lambda d\|_{L^{2}}^{1 / 2}\left\|\Lambda^{4} d\right\|_{L^{2}}^{3 / 2} \\
& \leq \frac{1}{8}\left\|\Lambda^{5} u\right\|_{L^{2}}^{2}+C\left\|\Lambda^{2} u\right\|_{L^{2}}^{2 / 3}\left\|\Lambda^{4} d\right\|_{L^{2}}^{2}, \\
\left|J_{33}\right| & \leq C\left\|\Lambda^{2} u\right\|_{L^{4}}\left\|\Lambda^{3} d\right\|_{L^{4}}\left\|\Lambda^{4} d\right\|_{L^{2}} \\
& \leq C\left\|\Lambda^{3} u\right\|_{L^{2}}^{5 / 6}\|u\|_{L^{2}}^{1 / 6}\|\Lambda d\|_{L^{2}}^{1 / 6}\left\|\Lambda^{4} d\right\|_{L^{2}}^{11 / 6} \\
& \leq C\left\|\Lambda^{3} u\right\|_{L^{2}}^{5 / 6}\left\|\Lambda^{4} d\right\|_{L^{2}}^{11 / 6}, \\
& \left|J_{34}\right| \leq C\|\nabla u\|_{L^{\infty}}\left\|\Lambda^{4} d\right\|_{L^{2}}^{2} .
\end{aligned}
$$

The estimate for $J_{4}$ is as follows:

$$
\begin{aligned}
\left|J_{4}\right|= & \left\|\Lambda^{4} d\right\|_{L^{2}}^{2}-\int_{\mathbb{R}^{2}} \Lambda^{4}\left(|d|^{2} d\right) \Lambda^{4} d \\
\leq & \left\|\Lambda^{4} d\right\|_{L^{2}}^{2}-3 \int_{\mathbb{R}^{2}}|d|^{2}\left|\Lambda^{4} d\right|^{2} \\
& +C \int_{\mathbb{R}^{2}}|d||\Lambda d|\left|\Lambda^{3} d\right|\left|\Lambda^{4} d\right| \\
& +C \int_{\mathbb{R}^{2}}|d|\left|\Lambda^{2} d\right|^{2}\left|\Lambda^{4} d\right| \\
& +C \int_{\mathbb{R}^{2}}|\Lambda d|^{2}\left|\Lambda^{2} d\right|\left|\Lambda^{4} d\right| \\
=: & \left\|\Lambda^{4} d\right\|_{L^{2}}^{2}-3\left\|d \Lambda^{4} d\right\|_{L^{2}}^{2}+J_{41}+J_{42}+J_{43} .
\end{aligned}
$$

We calculate $J_{41}, J_{42}$, and $J_{43}$ :

$$
\begin{aligned}
\left|J_{41}\right| & \leq C\left\|\Lambda^{3} d\right\|_{L^{4}}\|\Lambda d\|_{L^{4}}\left\|d \Lambda^{4} d\right\|_{L^{2}} \\
& \leq C\|\Lambda d\|_{L^{2}}^{1 / 6}\left\|\Lambda^{4} d\right\|_{L^{2}}^{5 / 6}\|\Lambda d\|_{L^{2}}^{5 / 6}\left\|\Lambda^{4} d\right\|_{L^{2}}^{1 / 6}\left\|d \Lambda^{4} d\right\|_{L^{2}} \\
& \leq C\left\|\Lambda^{4} d\right\|_{L^{2}}^{2}+\frac{3}{2}\left\|d \Lambda^{4} d\right\|_{L^{2}}^{2},
\end{aligned}
$$




$$
\begin{aligned}
\left|J_{42}\right| & \leq C\left\|\Lambda^{2} d\right\|_{L^{4}}^{2}\left\|d \Lambda^{4} d\right\|_{L^{2}} \\
& \leq C\|\Lambda d\|_{L^{2}}\left\|\Lambda^{4} d\right\|_{L^{2}}\left\|d \Lambda^{4} d\right\|_{L^{2}} \\
& \leq C\left\|\Lambda^{4} d\right\|_{L^{2}}^{2}+\frac{3}{2}\left\|d \Lambda^{4} d\right\|_{L^{2}}^{2}, \\
\left|J_{43}\right| & \leq C\left\|\Lambda^{2} d\right\|_{L^{4}}\|\Lambda d\|_{L^{8}}^{2}\left\|\Lambda^{4} d\right\|_{L^{2}} \\
& \leq C\|\Lambda d\|_{L^{2}}^{1 / 2}\left\|\Lambda^{4} d\right\|_{L^{2}}^{1 / 2}\|\Lambda d\|_{L^{2}}^{3 / 2}\left\|\Lambda^{4} d\right\|_{L^{2}}^{1 / 2}\left\|\Lambda^{4} d\right\|_{L^{2}} \\
& \leq C\left\|\Lambda^{4} d\right\|_{L^{2}}^{2} .
\end{aligned}
$$

Combining $J_{41}, J_{42}$, and $J_{43}$, we get

$$
J_{4} \leq C\left\|\Lambda^{4} d\right\|_{L^{2}}^{2} .
$$

Combining the above estimates to (20), we get

$$
\begin{gathered}
\frac{d}{d t}\left(\left\|\Lambda^{3} u\right\|_{L^{2}}^{2}+\left\|\Lambda^{4} d\right\|_{L^{2}}^{2}\right)+\left\|\Lambda^{5} u\right\|_{L^{2}}^{2} \\
\leq C\left(1+\|\nabla u\|_{L^{\infty}}+\left\|\Lambda^{2} u\right\|_{L^{2}}\right)\left\|\Lambda^{4} d\right\|_{L^{2}}^{2} \\
+C\left(1+\left\|\Lambda^{3} u\right\|_{L^{2}}\right)\left\|\Lambda^{4} d\right\|_{L^{2}}^{11 / 6} .
\end{gathered}
$$

Now we estimate the term $\int_{T_{0}}^{t}\left\|\Lambda^{3} u\right\|_{L^{2}}$ by applying the Gronwall inequality to (12):

$$
\begin{aligned}
\int_{T_{0}}^{t}\left\|\Lambda^{3} u\right\|_{L^{2}}^{2}(\cdot, \tau) d \tau \leq & \|\nabla u\|_{L^{2}}^{2}+\|\Delta d\|_{L^{2}}^{2} \\
& +\int_{T_{0}}^{t}\|\Delta \nabla u\|_{L^{2}}^{2}(\cdot, \tau) d \tau \\
\leq & \left(\left\|\nabla u_{0}\right\|_{L^{2}}^{2}+\left\|\Delta d_{0}\right\|_{L^{2}}^{2}\right) \\
& \times \exp \left(C \int_{T_{0}}^{t} 1+\|\nabla u\|_{L^{\infty}}(\cdot, \tau) d \tau\right) .
\end{aligned}
$$

Here $T_{0} \in(0, T)$ will be fixed later and we denote $\nabla u_{0}:=$ $\nabla u\left(\cdot, T_{0}\right), \Delta d_{0}:=\Delta d\left(\cdot, T_{0}\right)$. Set $A(t):=\max _{\tau \in\left(T_{0}, t\right)}\left(\|u\|_{H^{3}}^{2}+\right.$ $\left.\|d\|_{H^{4}}^{2}\right)(\tau)$. Now applying the logarithmic inequality [14]

$$
\|\nabla u\|_{L^{\infty}} \leq C\left(1+\|\nabla u\|_{\mathrm{BMO}}\left(1+\ln \left(1+\|u\|_{H^{3}}^{2}\right)\right)\right),
$$

we get

$$
\begin{aligned}
& \int_{T_{0}}^{t}\left\|\Lambda^{3} u\right\|_{L^{2}}^{2}(\cdot, \tau) d \tau \\
& \quad \leq C\left(T_{0}\right) \exp \left(C \int_{T_{0}}^{t}\|\nabla u\|_{L^{\infty}}(\cdot, \tau) d \tau\right)
\end{aligned}
$$

$$
\begin{aligned}
& \leq C\left(T_{0}\right) \exp \left(C \int_{T_{0}}^{t} 1+\|\nabla u\|_{\mathrm{BMO}}\right. \\
& \left.\times\left(1+\ln \left(1+\|u\|_{H^{3}}^{2}\right)\right)(\cdot, \tau) d \tau\right) \\
& \leq C\left(T_{0}\right) \exp \left(C \int_{T_{0}}^{t}\|\nabla u\|_{\mathrm{BMO}}(\cdot, \tau)\right. \\
& \quad \times(1+\ln (1+A(t))) d \tau) \\
& \leq C\left(T_{0}\right) \exp \left(C \int_{T_{0}}^{t}\|\nabla u\|_{\mathrm{BMO}}(\cdot, \tau) d \tau\right. \\
& \times(1+\ln (1+A(t)))) .
\end{aligned}
$$

Since $\|\nabla u\|_{\text {BMO }} \in L^{1}\left(T_{0}, T\right)$, we can take $T_{0}$ close enough to $T$, so that

$$
C \int_{T_{0}}^{t}\|\nabla u\|_{\mathrm{BMO}}(\cdot, \tau) d \tau \leq 2 \delta
$$

for some small positive number $\delta$ to be fixed later. With such choice of $T_{0}$ we have

$$
\int_{T_{0}}^{t}\left\|\Lambda^{3} u(\cdot, \tau)\right\|_{L^{2}}^{2} \tau \leq C\left(T_{0}\right)(1+A(t))^{2 \delta} .
$$

Hölder's inequality gives

$$
\int_{T_{0}}^{t}\left\|\Lambda^{3} u(\cdot, \tau)\right\|_{L^{2}} \tau \leq C\left(T_{0}\right)(1+A(t))^{\delta} .
$$

Fix $T_{0}$ satisfying

$$
C \int_{T_{0}}^{t}\|\nabla u(\cdot, \tau)\|_{\mathrm{BMO}} \tau \leq 2 \delta, \quad \ln \left(1+A\left(T_{0}\right)\right)>1 .
$$

Combining the above estimates together, we get

$$
\begin{aligned}
& \frac{d}{d t}\left(\|u\|_{H^{3}}^{2}+\|d\|_{H^{4}}^{2}\right) \\
& \leq C\left(1+\left\|\Lambda^{3} u\right\|_{L^{2}}\right) A(t)^{11 / 12} \\
& +\left(\|\nabla u\|_{L^{\infty}}+\left\|\nabla^{2} u\right\|_{L^{2}}+1\right) A(t) \\
& \leq C\left[1+\|\nabla u\|_{\mathrm{BMO}}(1+\ln (1+A(t)))\right. \\
& \left.\quad+\left\|\nabla^{2} u\right\|_{L^{2}}\right] A(t)+C\left(1+\left\|\Lambda^{3} u\right\|_{L^{2}}\right) A(t)^{11 / 12} \\
& \leq C\left(T_{0}\right)\left[\left(\|\nabla u\|_{\mathrm{BMO}}+\left\|\nabla^{2} u\right\|_{L^{2}}+1\right) A(t)\right. \\
& \left.\quad \times \ln (1+A(t))+\left(1+\left\|\Lambda^{3} u\right\|_{L^{2}}\right) A(t)^{11 / 12}\right] .
\end{aligned}
$$


Integrating the above inequality, we have

$$
\begin{gathered}
A(t) \leq C\left(T_{0}\right) A_{0}+C\left(T_{0}\right) \int_{T_{0}}^{t} 1+\left\|\Lambda^{3} u\right\|_{L^{2}}(\cdot, \tau) d \tau A(t)^{11 / 12} \\
+C\left(T_{0}\right) \int_{T_{0}}^{t}\left(1+\|\nabla u\|_{\mathrm{BMO}}+\left\|\Lambda^{2} u\right\|_{L^{2}}\right) A(t) \\
\times \ln (1+A(t)) d \tau
\end{gathered}
$$

where $A_{0}:=\|u\|_{H^{3}}^{2}\left(T_{0}\right)+\|d\|_{H^{4}}^{2}\left(T_{0}\right)$.

Taking $\delta=1 / 24$, we have

$$
\int_{T_{0}}^{t} 1+\left\|\Lambda^{3} u\right\|_{L^{2}} d \tau \leq C\left(T_{0}\right)(1+A(t))^{1 / 24} .
$$

Thus (35) tells us that

$$
\begin{aligned}
& A(t) \leq C\left(T_{0}\right) A_{0}+C\left(T_{0}\right)(A(t)+1)^{1 / 24} A(t)^{11 / 12} \\
&+C\left(T_{0}\right) \int_{T_{0}}^{t}\left(1+\|\nabla u\|_{\mathrm{BMO}}+\left\|\Lambda^{2} u\right\|_{L^{2}}\right) A(t) \\
& \times \ln (1+A(t)) d \tau .
\end{aligned}
$$

This in turn gives

$$
\begin{aligned}
1+A(t) \leq & C\left(T_{0}\right)\left(1+A_{0}\right)+C\left(T_{0}\right)(A(t)+1)^{23 / 24} \\
+ & C\left(T_{0}\right) \int_{T_{0}}^{t}\left(1+\|\nabla u\|_{\mathrm{BMO}}+\left\|\Lambda^{2} u\right\|_{L^{2}}\right) \\
& \times(A(t)+1) \ln (1+A(t)) d \tau .
\end{aligned}
$$

We set $B(t):=(1+A(t))^{1 / 24}, B_{0}:=\left(1+A_{0}\right)^{1 / 24}$ and divide the above inequality by $(1+A(t))^{23 / 24}$; using the monotonicity of $A(t)$ we reach

$$
\begin{aligned}
B(t) \leq C\left(T_{0}\right)\left[B_{0}+1+\int_{T_{0}}^{t}(1\right. & \left.+\|\nabla u\|_{\mathrm{BMO}}+\left\|\nabla^{2} u\right\|_{L^{2}}\right) \\
& \times B(t) \ln B(t) d \tau] .
\end{aligned}
$$

The standard Gronwall's inequality now gives

$$
B(t) \leq\left[C\left(T_{0}\right)\left(1+B_{0}\right)\right]^{\exp \left[C\left(T_{0}\right) \int_{T_{0}}^{t} 1+\|\nabla u\|_{\mathrm{BMO}}+\left\|\Lambda^{2} u\right\|_{L^{2}} d \tau\right]},
$$

which leads to

$$
A(t) \leq\left[C\left(T_{0}\right)\left(1+B_{0}\right)\right]^{24 \exp \left[C\left(T_{0}\right) \int_{T_{0}}^{t} 1+\|\nabla u\|_{\mathrm{BMO}}+\left\|\Lambda^{2} u\right\|_{L^{2}} d \tau\right]} .
$$

As $\int_{T_{0}}^{t}\|\nabla u\|_{\mathrm{BMO}}+\left\|\Lambda^{2} u\right\|_{L^{2}} d \tau$ remains bounded as $t \nearrow T$, the above inequality contradicts that $A(t) \nearrow \infty$ as $t \nearrow T$, so we complete our proof of Theorem 1.

\section{Conflict of Interests}

The author declares that there is no conflict of interests regarding the publication of this paper.

\section{References}

[1] D. Coutand and S. Shkoller, "Well-posedness of the full Ericksen-Leslie model of nematic liquid crystals," Comptes Rendus de l'Académie des Sciences I. Mathématique, vol. 333, no. 10, pp. 919-924, 2001.

[2] F. H. Lin and C. Liu, "Nonparabolic dissipative systems modeling the flow of liquid crystals," Communications on Pure and Applied Mathematics, vol. 48, no. 5, pp. 501-537, 1995.

[3] F. H. Lin and C. Liu, "Existence of solutions for the EricksenLeslie system," Archive for Rational Mechanics and Analysis, vol. 154, no. 2, pp. 135-156, 2000.

[4] J. Fan, G. Nakamura, and Y. Zhou, "Global regularity for the 2D liquid crystal model with mixed partial viscosity".

[5] J. Fan and T. Ozawa, "Regularity criterion for the incompressible viscoelastic fluid system," Houston Journal of Mathematics, vol. 37, no. 2, pp. 627-636, 2011.

[6] C. Cao, J. Wu, and B. Yuan, "The 2D incompressible magnetohydrodynamics equations with only magnetic diffusion," SIAM Journal on Mathematical Analysis, vol. 46, no. 1, pp. 588-602, 2014.

[7] C. V. Tran, X. Yu, and Z. Zhai, "On global regularity of 2D generalized magnetohydrodynamic equations," Journal of Differential Equations, vol. 254, no. 10, pp. 4194-4216, 2013.

[8] J. Fan, G. Nakamura, and Y. Zhou, "Global Cauchy problem of 2D generalized MHD equations".

[9] Q. Jiu and J. Zhao, "A remark on global regularity of 2D generalized magnetohydrodynamic equations," Journal of Mathematical Analysis and Applications, vol. 412, no. 1, pp. 478-484, 2014.

[10] J. Wu, "Global regularity for a class of generalized magnetohydrodynamic equations," Journal of Mathematical Fluid Mechanics, vol. 13, no. 2, pp. 295-305, 2011.

[11] Y. Wang, A. Alsaedi, T. Hayat, Z. Jiang, and Y. Zhou, "Global regularity for the incompressible $2 \mathrm{D}$ generalized liquid crystal model with fractional diffusions," Applied Mathematics Letters, 2014.

[12] T. Kato and G. Ponce, "Commutator estimates and the Euler and Navier-Stokes equations," Communications on Pure and Applied Mathematics, vol. 41, no. 7, pp. 891-907, 1988.

[13] C. E. Kenig, G. Ponce, and L. Vega, "Well-posedness of the initial value problem for the Korteweg-de Vries equation," Journal of the American Mathematical Society, vol. 4, no. 2, pp. 323-347, 1991.

[14] H. Kozono and Y. Taniuchi, "Limiting case of the Sobolev inequality in BMO, with application to the Euler equations," Communications in Mathematical Physics, vol. 214, no. 1, pp. 191200, 2000 . 


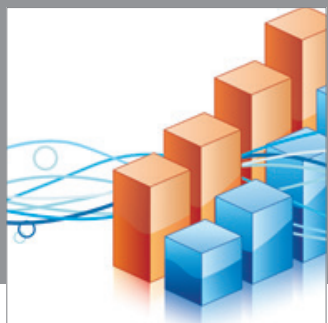

Advances in

Operations Research

mansans

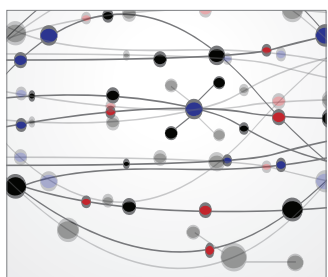

The Scientific World Journal
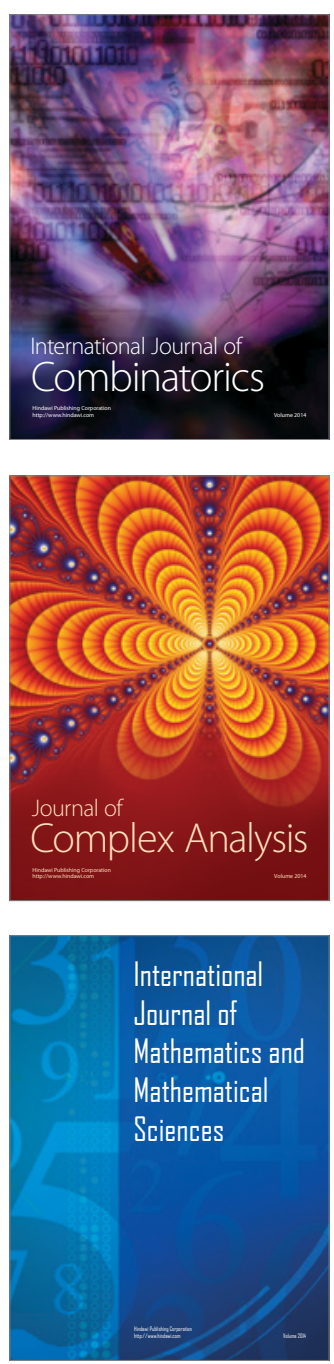
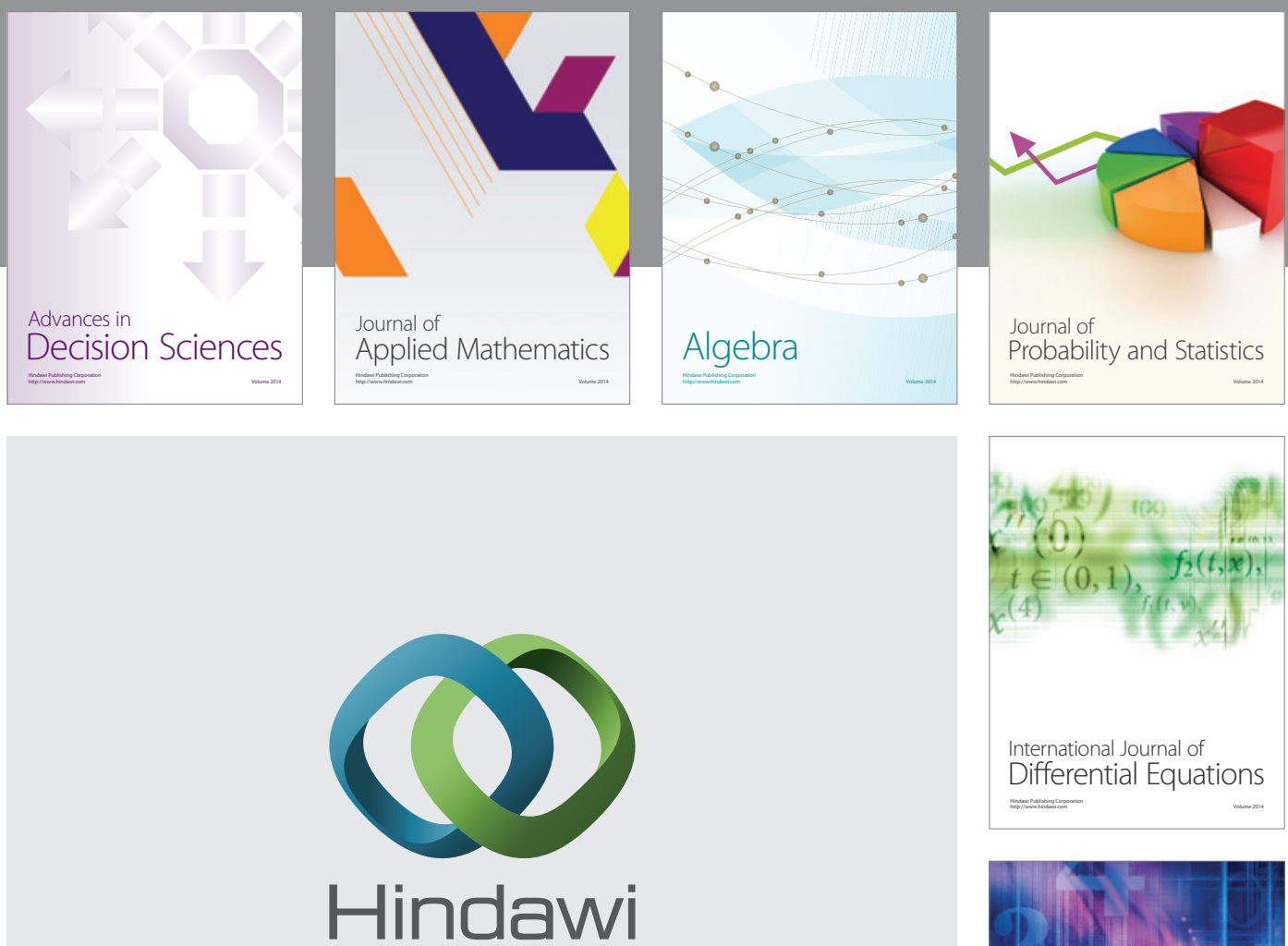

Submit your manuscripts at http://www.hindawi.com
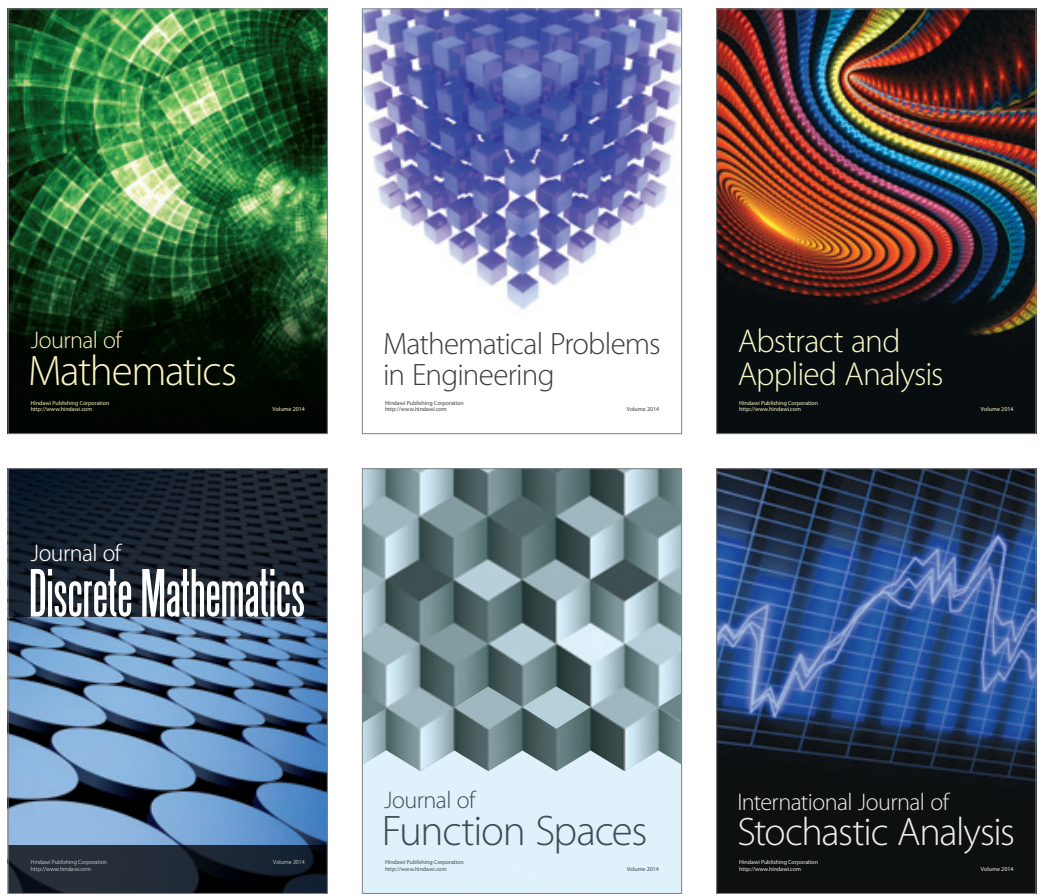

Journal of

Function Spaces

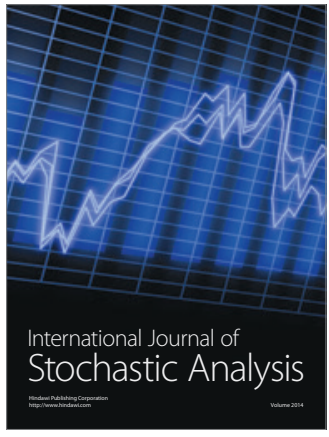

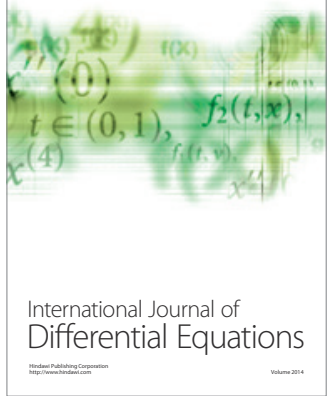
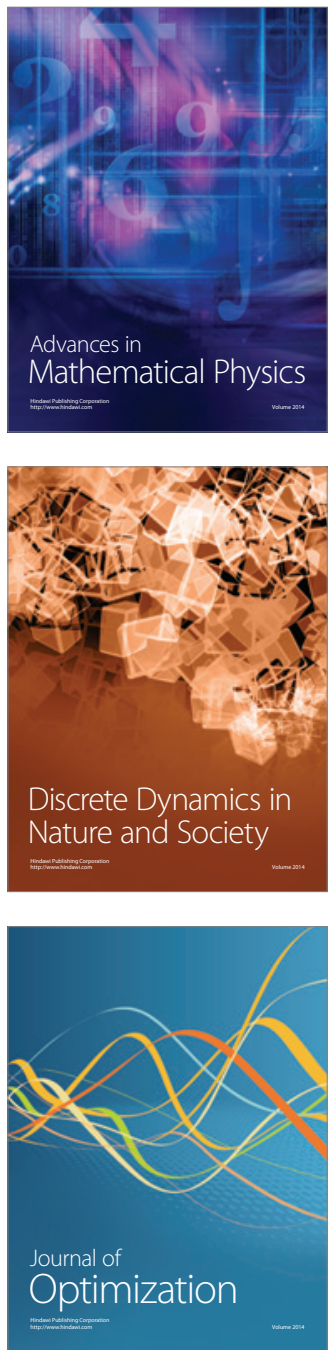